\title{
CONFERENCE REPORT International Symposium on Multiple Sclerosis University of Western Ontario May 3-5, 1981
}

\author{
S. DALES \\ G. EBERS \\ T. FEASBY \\ D. PATY
}

A three day symposium concentrating mainly on the research aspects of multiple sclerosis was held under the auspices of the Multiple Sclerosis Society of Canada, and The University of Western Ontario, Department of Clinical Neurological Sciences. The first day was devoted to neurovirological studies in tissue culture and in experimental animals. The concepts of viral latency in the human were also examined. The second day dealt with immunology, principally immune regulation as it applies to experimental models of immunological brain disease and multiple sclerosis (MS). In the evening a poster session was held with a work-shop discussion related to poster displays and issues raised during the first two days. The third day included a discussion of the physiology of demyelination and the use of laboratory tests in the diagnosis of MS, as well as general concepts of management.

\section{Neurovirological Studies}

Dr. Clarence Gibbs (National Institutes of Health, U.S.A.) opened the meeting with a discussion of a new medium for tissue culture for normal neuronal and other central nervous system cellular elements. This new medium contains $20 \%$ serum supplemented by insulin and glucose, and enhances growth of large numbers of neurons and axonal processes. Appearance in the cultures of neuronal and glial elements suggests that normal differentiation occurs in vitro. Dr.
Gibbs and his colleagues have recently found that serum from patients with Cretuzfeld Jakob (CJ) disease specifically binds to "neuroaxonal filaments" presumably due to the presence of antibody against a neurofilament protein. Binding occurred with $48 \%$ of transmittable CJ disease, $24 \%$ of Kuru patients' sera, $11 \%$ of sera from other neurological diseases, and $8 \%$ of control sera. The antibody was not cytotoxic. It is not known whether specific antibody is evoked as a primary event, or a secondary one arising from tissue breakdown. The same antibody binding was found to occur with sera from primates that had been inoculated in transmission studies of Kuru and $\mathrm{CJ}$ disease.

Dr. Ashley Haase (University of California, San Francisco) presented data on Visna and measles virus using nucleic acid in-situ hybridization and autoradiography for the localization of viral genome in brain cells. This procedure can detect latent genome in the absence of antigenic expression. Samples of CNS from four patients with MS have been examined by insitu hybridization with a nucleic acid probe for measles virus. Material from one of these MS patients was positive. Additional studies are being made using samples from both MS and normal brains.

Dr. Marian Coulter (University of Western Ontario) presented studies of viral persistence in cultured glial cells. The surprising findings are that RNA viruses of the corona and measles families can establish prolonged latent infections in-vitro within cells of neural and other origin when the temperature is elevated to $40^{\circ} \mathrm{C}$. Restriction of virus expression during the latent phase is most probably controlled by a factor produced by the host cell, probably interferon.

Dr. John Subak-Sharpe (Institute of Virology, Glasgow, Scotland) described his work using restriction endonuclease enzymes for analyzing variability of the genome between isolates of Herpes Simplex Virus (HSV). Examination of HSV recovered from various human tissues revealed that a single person may carry persistent virus in several sites, each of which is identical in its HSV subtype DNA content. HSV isolated from different individuals may belong to one of 15-20 subgroups, as evident from differences in DNA sequence. These exciting findings suggest that HSV variability among individuals could account for the variable clinical expression in virus infections, possibly accounting for the differences in virulence often seen with these virus infections in humans.

Dr. Richard Barringer (University of California, San Francisco) reviewed the history of the recovery of HSV from human ganglia. He emphasized that the localization of $\mathrm{HSV}$ in the experimental model is specific to ganglia associated with the area of inoculation. He speculated that HSV encephalitis might arise by a transdural movement of the virus from 
the trigeminal ganglia to the temporal lobes.

Dr. Kenneth Warren (University of Alberta) presented his data on the recovery of $\mathrm{HSV}$ from normal human cadavers and one individual with MS. HSV has been recovered very frequently from trigeminal and other sensory ganglia. It is unusual to recover HSV from the brain, except in patients with acute HSV encephalitis. Dr. Warren has found that the recovery of latent $\mathrm{HSV}$ from the trigeminal and other ganglia is more successful when culture conditions allow the explant cell monolayers to develop over a large surface area.

$\mathrm{Mr}$. Ole Sorenson (University of Western Ontario) reported on an invivo study of demyelination in the rat secondary to mouse hepatitis virus (JHM) infection. He found evidence of progressive CNS demyelination during paralysis and remyelination in recovering animals. He was also able to ind uce late onset demyelination by the use of immunosuppression in animals which had survived the initial infection.

Dr. Erling Norrby (Karolinska Institute, Stockholm) described his studies on measles virus using monoclonal antibodies against the various peptides. The $\mathrm{M}$ protein (molecular weight 36,000 ) appears to be a peculiar antigen. He finds a consistent low antibody response to this $M$ protein in patients with SSPE and chronic active hepatitis.

Dr. Brian Summers (New York State College of Veterinary Medicine) presented his studies on the neuropathology and pathogenesis of distemper virus (CDV) in dogs. Intranasal inoculation with CDV in beagles produces primarily severe acute disease in young dogs and demyelination in older animals. In the first 5 days the virus goes to local lymphoid organs. It then circulates via lymphoid tissue and in seven days a mild encephalomyelitis appears. This may disappear in two weeks. If the infection becomes persistent demyelination occurs.

Optic neuritis can develop in CDV inoculated dogs. It is not unusual for them to develop bilateral blindness. If the dog survives for thirty days there is widespread demyelination which includes the periventricular regions. There is also astrocyte reactivity. Early in the disease there is usually noninflammatory demyelination; only later does an inflammatory component develop. These affected dogs contain high levels of interferon in the spinal fluid. Low cell-mediated responses are seen in all lethal infections. Dr. Summer's concept is that the infammation is anti-viral and does not specifically cause demyelination. He suggested that demyelination could be due to fusion between the macrophage and the myelin membrane resulting in secondary myelin destruction.

Dr. William Hall (Rockefeller University) presented results of an analysis of measles virus polypeptides. He found that a vigorous antibody response to the $M$ protein occurs in patients with atypical measles. Multiple Sclerosis patients developed a good response to the $M$ protein, but in contrast, patients with SSPE produced very little response in either serum or cerebrospinal fluid. He proposed that this defective production might be due to a host restriction of $M$ protein production rather than to an immunogolical deficit in antibody response. This mechanism could be seen as a brain specific restriction on viral growth.

Studies in patients with MS failed to show any evidence for persistance of measles or CDV antigens in brain. Hall also postulated that in chronic CNS disease one could have a generalized restriction on virus expression thereby abrogating the ability to find evidence of viral infection at a later date.

Dr. Hilary Koprowski (Wistar Institute, Philadelphia) reviewed his work with monoclonal antibodies in the study of persistent virus infections. The model system for this work has been rabies. The use of monoclonal antibodies to rabies virus has now shown remarkable variability in the antigenic properties of various rabies isolates. These strain differences could explain the failure of passive immunization to produce protection in some patients. Dr. Koprowski and his colleagues have also developed monoclonal reagents for the study of other viruses. A monoclonal anti-idotype antibody has been formed against one MS spinal fluid immunolglobulin. This antibody appears to be unique to that particular patient. The monoclonal antibody also reacts against the WW strain of Theiler's virus. He suggested that in the future monoclonal antibodies might be used as a means for specific therapy in viral infections, tumors, and leukemias.

\section{Immunological Studies}

Dr. Peter Doherty (Wistar Institute) began the second day with a review of the basic aspects of immune regulation. He recalled Jerne's analogy between the nervous system and the immune system. These are the two systems in the body which recognize foreign stimuli. The anatomy of the nervous system is stable in contrast to the anatomy of the immune system which must allow for very rapid adaptations in response to foreign antigens. The specificity of the nervous system is related to the nature of receptors and established pathways. Specificity in the immune system is due to specific binding sites on individual cells. Learning and memory in the nervous system are again related to established pathways, whereas learning and memory in the immune system are related to cell interactions and clonal expansion.

The main effector limb of the nervous system is through direct messages to motor neurons, whereas in the immune system the cells themselves and cell products are the effectors. Self-recognition is an essential part of both systems. This recognition is essential for the nervous system on a psychological level, whereas in the immune system, recognition of self plays an important role in regulation and in monitoring. Immune recognition occurs via idiotypic networks and the major histocompatibility complex. Control of the nervous system is both voluntary and involuntary, while control of the immune system is totally involuntary.

The idiotypic network was explained as a type of self-self interaction in order to regulate antibody production with the same principles applying to cell-cell interactions. The relationship between inducer cells, cytotoxic $\mathbf{T}$ 
cells, and cell mediated immunity was explained. While the idiotypic network functions via recognition of the hypervariable region of the immunoglobulin molecule, the $T$ cell is focused onto other cells via the major histocompatibility complex.

The entire system is seen as an "immunological orchestra" where the orchestral score is written with idiotypes, antigens, and the major histocompabibility complex. Using these notes as structure the various antibodies and cell components interact in order to complete the recognition and effector function of the system.

Dr. Robert Kibler(Emory University, Atlanta) presented work on myelin basic protein (MBP) and its fragments. The active encephalitogenic site for producing experimental allergic encephalomyelitis (EAE) also appears to be necessary to produce protection against EAE. He has been unable to protect animals using non-encephalitogenic portions of the molecule. He stressed the point made by Alvord and his colleagues that MBP for the treatment of MS has not yet received a fair trial. There also is a need to consider adjuvant therapy in addition to the use of MBP in the possible treatment of MS.

Dr. Philip Paterson (Northwestern University, Chicago) discussed his recent studies on EAE. He stressed that the clinical and histopathological manifestations of EAE may be due to two separate mechanisms. The early influx of fibrin and edema in the perivenular portions of the brain in EAE seems to correlate very well with the clinical signs. The histopathological features of EAE can be produced by the transfer of supernatants from lymph nodes of animals with EAE induced using whole spinal cord. He could not produce this phenomenon using myelin basic protein alone. The mechanism of this transfer remains unclear. $\mathrm{He}$ has recently been able to detect oligoclonal banding (OB) in the spinal fluid of animals with EAE. He was unable to absorb the $\mathrm{OB}$ from CSF using spinal cord or MBP. He was also unable to correlate the antiMBP activity in the $\operatorname{lgG}$ fraction with the oligoclonal bands. His studies of endogenous circulating MBP suggest that blood levels of MBP may be a good predictor of recovery from EAE. Detectible MBP falls at the time of appearance of high affinity anti-MBP antibody. In these animals free serum MBP again appears coincident with recovery. He emphasized that normal humans have detectable amounts of circulating MBP in the serum and perhaps under normal circumstances this has an immunoregulatory function. He also proposed that perhaps two different subsets of immune competent cells are important in producing clinical signs on one hand, and pathological findings on the other.

Dr. Gil Strejan (University of Western Ontario) presented data on the use of MBP coupled to liposomes in the prevention of EAE. Liposomes work effectively as adjuvant to prevent the development of EAE and the MBP liposome combination is more active than the use of soluble MBP alone in producing suppression.

Dr. C.F.C. MacPherson (University of Western Ontario) reviewed her work on the use of spinal cord protein (SCP) to protect against EAE. She has been able to extract this protein from both peripheral nerve (cross reacting with P-2 protein) and from the central nervous system. Both proteins will protect animals against the development of EAE. She proposed that such a naturally occuring substance might possibly be useful in the treatment of human demyelinating disease.

Dr. Dale McFarlin (National Institutes of the Health, U.S.A.) presented his recent work in the production of EAE in the mouse. He emphasized that in some situations, susceptibility to EAE is due to a preprimed immunological orchestra, and resistance could well be due to a specifically subdued one. A new modulation of the protocol for production of EAE in SJL the mouse was presented. This employs in-vitro boosting of sensitized lymphocytes prior to adoptive transfer. This model should allow more specific studies of immune regulation in EAE in this susceptible strain.

Dr. William Marshall (Memorial University, Newfoundland) reviewed in general terms the major histocom- pability complex of man as well as his own studies of complement alleles. $\mathrm{He}$ proposed that a mutation in one individual in the distant past could have increased susceptibility to MS in the descendants of that individual. After one thousand years of evolution, the susceptibility haplotypes could remain intact in $50 \%$ of the descendants.

Dr. Jack Antel (University of Chicago) discussed supressor cell mechanisms in man, specifically in MS. Until recently, functional assays were employed using mitogen stimulated lymphocytes to suppress other cellular functions such as cell proliferation or antibody production. Current studies have employed monoclonal antibodies which react with $T$ cell surface antigens. Specific subsets of $T$ cells can be identified by these antigens allowing quantitation of $\mathrm{T}$ helper and $T$ supressor lymphocytes. He presented a theory to account for the loss of $\mathrm{T}$ suppressor cells in MS. Sharing of antigens between oligodendrocytes and $T$ supressor cells could explain a general (secondary) drop in supressor cells associated with the acute relapse. A cytotoxic cell or antibody directed toward oligodendrocytes and supressor $T$ cells could at the same time damage the nervous system and supressor cells. He pointed out that this is a new and rapidly moving field. Not all laboratories have been able to confirm a fall in supressor cell activity in the acute relapse.

Dr. George Ebers (University of Western Ontario) presented preliminary results of a large population-based study of MS genetics carried out at the MS Clinic in London, Ontario. Over 600 individuals from 60 multiplex MS families were examined and typed for HLA-A, B, C, Dr, C2, C4, and BF. Preliminary results which were especially informative concerned 40 unequivocally typed MS sibling pairs. Ten pairs shared no HLA antigens. These findings differed significantly from studies of pooled data in the literature. Genetic heterogeneity or selection bias were considered possible explanations for the discrepancy. He concluded that on the basis of the available evidence, HLA linked factors play only a minor role in overall MS susceptibility. 
Dr. Bodvar Vandvik (Oslo, Norway) discussed his studies of antibody responses in the central nervous system secondary to viral infections. Oligoclonal banding (OB) has been seen in a number of viral infections of the nervous system of which SSPE has been the most characteristic. $40 \%$ of patients with mumps meningitis have been found to have oligoclonal banding. These patients also appear to have specific antibody responses to measles, rubella, and herpes simplex virus in their CSF. These findings show a non-specific response in antibody production during the course of a specific viral infection. It is not unusual to see the pattern of these antibody bands change over a period of time in acute viral infections. In contrast to this, the OB pattern in MS tends to remain constant over many years.

Dr. Vandvik pointed out that the use of isoelectric focusing is an extremely sensitive test for the identification of these antibody bands. It is so sensitive that apparent bands can be detected in normals. He has been unable to identify the antigen specificities of the major spinal fluid bands found in MS. In contrast, the antigenic specificities of many of the bands found in viral CNS infections are specific for the antigens of the infecting virus. The additional bands seen in viral meningitis can usually be identified as having other viral specificities.

Dr. George Ebers presented his studies of idiotypic and anti-idiotypic (id) antibodies in the study of MS CSF bands. He has been successful in producing in rabbits a number of antiidiotypic sera against these bands. Major MS bands do not appear to contain cross-idiotypic specificities. Anti-id sera were used to detect idiotype in other bands from the same patient and to measure idiotype levels in serum and CSF. The antisera made possible the serial determination of the idiotype in the same patient as well as quantitation of idiotype from several plaques taken from one autopsy case. He found that idiotype levels tended to remain the same over time. He suggested that because anti-idiotypes may simulate antigens, such reagents may be of some special value in determining the relevance of oligoclonal bands to the pathogenesis of MS.

Dr. Byron Waksman of the National Multiple Sclerosis Society of the United States summed up the two days of virology and immunology by emphasizing that we should continue to think of viral agents as triggers of the MS process. Recent technological breakthroughs in virology and immunology promise further important advances in knowledge in these fields over the next few years. Animal models of demyelination including EAE and JHM virus infection should contribute to our understanding of the mechanisms of demyelination. It is exciting that the SJL mouse can now be used for the study of relapsing and remitting EAE. This will allow specific study of the defects and control of immune regulation in this model. Antigens other than myelin basic protein should be evaluated in EAE. He challenged the workers in EAE to use whole oligodendrocytes to produce the disease. The presence of antibodies in the spinal fluid of these animals must be investigated in order to determine the responsible antigen. $\mathrm{He}$ suggested that the ongoing disease process may depend on the involvement of secondary antigens.

Dr. Waksman pointed to a number of studies involving MS patients which have been promising but need further clarification. These include the HLA relationships to susceptibility, the recently described point epidemics and the appearance of activated lymphocytes in the blood and spinal fluid of MS patients (he suggested caution here because cells from MS patients apparently are sticky and can easily adhere to central nervous system and target cells). Other observations which require full investigation include the findings of immunoregulatory changes in MS patients, the presence of shared antigens on oligodendrocytes and $T$ suppressor cells, the reported deficiency in interferon production, the deficiency of natural killer cells in MS patients, and the presence of large numbers of plasma cells in MS lesions. He emphasized that these are exciting times in MS research and that major advances in the understanding of the pathogenesis and pathophysiology of this disorder could be expected over the next few years.

\section{Diagnosis and Treatment}

This section of the symposium was opened by Professor Tom Sears, Institute of Neurology, London, U.K., who reviewed the pathophysiology of demyelination. He indicated that conduction block, the most serious physiologic consequence of demyelination, can be potentially corrected by increasing the duration of the action potential. This has been achieved experimentally by cooling and by using toxins which interfere with the mechanism for inactivation of sodium channels. The recent finding that potassium channels occur in demyelinated axons but not at normal nodes facilitated the discovery that potassium channel blockers, when applied to demyelinated axons, could prolong the action potential and thereby overcome conduction block. Further progress in this work may lead to the development of clinically useful pharmacologic agents in MS.

Dr. Terry Picton (University of Ottawa) discussed the theoretical and technical aspects of evoked potentials (EP). Dr. Andrew Eisen (University of British Columbia) presented his work on somatosensory evoked potentials in central nervous system (CNS) disease. He showed that the CNS amplifies the input received from the periphery. Dr. William Brown (University of Western Ontario) presented his work on flashevoked blink reflexes. This is an inexpensive and technically easy method for the identification of clinically silent CNS lesions in MS. Dr. Tom Feasby (University of Western Ontario) presented the results of evoked potential studies in 184 patients with "definite" MS. AEP's, VEPs and blink reflexes were each abnormal in $30 \%$ of patients whose disease was less than 3 years duration. They were abnormal in $60 \%$ to $80 \%$ of patients whose disease was greater than 10 years in duration. In cases of "possible" MS, "new" lesions were found in $24 \%$ of patients using AEP or VEP and $33 \%$ of patients using blink reflexes. In acute unilat- 
eral optic neuritis, electrophysiological abnormalities were found in the asymptomatic eye in 13 of 27 cases. In this group of "possible" MS, electrophysiological studies showed evidence of dissemination in $48 \%$.

Doctors George Ebers and Bodvar Vandvik, discussed the usefulness of cerebrospinal fluid in the diagnosis of MS. Dr. Ebers pointed out that agarose electrophoresis of concentrated (x100) cerebrospinal fluid could detect oligoclonal banding in up to $95 \%$ of patients with clinically definite MS. Eight percent of 500 non-MS patients had oligoclonal banding. Half of these were patients with non-immune disorders of the nervous systems. This means that 4 out of 100 patients with miscellaneous disorders not thought to be due to an immune mechanism could have oligoclonal banding. This is contrasted with a $95 \%$ abnormality rate in patients known to have MS. This degree of sensitivity and specificity closely approaches that of laboratory tests in other diseases which are considered to be "diagnostic".

The bands consist mainly of immunoglobulin G. Serum electrophoresis must always be done for comparison. The concentration of immunoglobulin $G$ in the electrophoresed sample should normally be the same for both serum and cerebrospinal fluid studies. Isoelectric focusing (IEF) may be too sensitive a method for use in routine diagnosis. Doctors Ebers and Vand vik showed evidence that post-synthetic changes on a single immunoglobulin can produce multiple bands using IEF. "Bands" may also occur in pooled $\mathrm{IgG}$ as an artifact of the method in isoelectric focusing whereas it is unlikely to be seen as an artefact in agarose electrophoresis.

Dr. Fernando Vinuela (University of Western Ontario) reported his experience in the use of CT scanning in MS. With a large volume of contrast ( $80 \mathrm{mg}$ of iodine) and a scan delayed by one hour, it is common to see multiple areas of abnormality in the cerebral hemispheres, particularly during acute phases of MS.

Dr. John Kurtzke (Georgetown University, Washington, D.C.) reviewed the natural history and epidemiology of MS. He summarized the data from
200 epidemiological studies in the literature to demonstrate the worldwide geographic distribution of MS. The prevalence of MS varies by as much as a factor of six in some closely related geographic areas in Europe, especially in Switzerland. A review of MS in veterans of World War II and the Korean War, shows that MS continues to be more prevalent in the northerly portions of the United States.

Recent studies have indicated that there have been small epidemics of MS in both the Faroes and Iceland over the last four decades. Age and year of onset correlations suggest a relationship between the presence of British troops during World War II and the subsequent development of MS in the local population. This raises the possibility that MS is a communicable disease, at least in these special circumstances.

Many of the epidemiologic studies have suggested that some environmental factor acting around the time of puberty plays a role in the subsequent development of MS. Kurtzke urged virologists to continue their efforts to identify a virus which might be the initiating factor in MS.

A panel discussion was held on diagnostic criteria in MS. Dr. Charles Poser (University of Vermont) introduced the topic by cautioning the audience that many tests such as CT scanning, HLA typing, evoked potentials, and CSF analysis are used indiscriminately for the diagnosis of MS. The costs of these tests can be enormous. Poser stated that the most useful non-invasive diagnostic aids were evoked potentials. $\mathrm{He}$ also mentioned the usefulnesss of the hot bath test and evaluation of vision with colour charts.

Dr. Donald Paty (University of British Columbia) pointed out that many of the physiological tests developed in recent years to aid in the diagnosis of MS are reliable indicators of subclinical lesions and are now widely used in routine neurological practice. In addition, the presence of oligoclonal banding in the CSF probably has a single test reliability of $85 \%$ in diagnosing MS. The currently accepted diagnostic criteria (Schum- acher 1965$)$ are probably too exclusive. Paty and his colleagues at the University of Western Ontario have proposed a revision of the Schumacher criteria. The basic criteria for age, duration of symptoms and clinical evidence of white matter lesions would continue to apply, but it was proposed that the following laboratory abnormalities be included as signs of dissemination of lesions in space.

(1) Prolonged VEP latency $>3$ SD over the mean) with typical wave form.

(2) Prolonged I-III or III-V Latencies (>3SD) on ABER testing.

(3) Prolonged SSEP $(>3 S D)$ with typical wave forms. Amplitude changes alone should not be accepted as evidence of an evoked potential abnormality.

It was also proposed the oligoclonal banding (OB) on argarose electrophoresis of concentrated CSF be accepted as a sign of CNS inflammation highly suggestive of MS. This would then allow inclusion of a category of Laboratory Probable MS under the following circumstances:

(1) A single attack with clinical evidence of multiple typical lesions disseminated in space plus oligoclonal banding.

(2) A single attack with clinical evidence of a typical lesion at one site plus an evoked potential abnormality indicating a lesion at another site plus oligoclonal banding.

(3) Recurrent attacks suggesting a typical lesion at one site plus oligoclonal banding.

Dr. Peter Ashby (University of Toronto) discussed management of spasticity. He pointed out that relief of spasticity may not always be beneficial to patients with MS. Patients most likely to be benefited by drug therapy are those that are bedridden, or those that are fully ambulatory but disabled by spasticity. He reviewed the use of Baclofen, Diazepam, and Dantrolene.

Dr. William Sibley closed the meeting with a discussion of treatment in MS, focusing on efforts designed to influence the natural history of the disease. He pointed out that recent epidemiological studies have indicated that after 20 years $78 \%$ of patients with 
MS a re still independent and working. The only proven therapy for the acute relapse in MS has been ACTH. Future treatment trials must be carried out with assessment by patient selfevaluation, standard neurological examination, quantitative neurological examination, evoked potentials, cerebrospinal fluid findings, and computerized tomography. He noted the problem of dependency in patients who have been treated over a long period of time with corticosteroids and the very high complication rate with use of massive immunosuppression.
It was the feeling of many of the participants who are involved in MS related research that study of antigen specific systems of immune regulation in this disorder could eventually point toward specific methods for immune modulation. Studies of idiotypic antibodies and the search for the specific antigen responsible for the autoimmune process could allow specific manipulation of the immune system in such a way as to maintain a spontaneous remission once it has occurred. The relapsing and remitting form of MS that is seen in at least $70 \%$ of patients during the first few years, provides an unique opportunity to study changes in the immune system that occur during the spontaneous remission. Understanding of these changes could well lead to a specific therapy directed toward inducing and maintaining remission.

\section{ACKNOWLEDGEMENTS}

This symposium was supported by the University of Western Ontario, the Multiple Sclerosis Society of Canada, the Fogarty International Centre of the National Institutes of Health, the Royal College of Physicians and Surgeons of Canada and the Ciba Corporation. 